\title{
Appareil
}

$10 \mid 2012$

Lyotard et la surface d'inscription numérique

\section{Les Immatériaux}

\section{Marta Hernandez}

\section{OpenEdition}

Journals

Édition électronique

URL : http://journals.openedition.org/appareil/93

DOI : 10.4000/appareil.93

ISSN : 2101-0714

Éditeur

MSH Paris Nord

Référence électronique

Marta Hernandez, «Les Immatériaux », Appareil [En ligne], 10 | 2012, mis en ligne le 01 février 2008 , consulté le 30 juillet 2020. URL : http://journals.openedition.org/appareil/93 ; DOI : https://doi.org/ 10.4000/appareil.93

Ce document a été généré automatiquement le 30 juillet 2020.

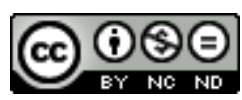

Appareil est mis à disposition selon les termes de la Licence Creative Commons Attribution - Pas d'Utilisation Commerciale - Pas de Modification 4.0 International. 


\title{
Les Immatériaux
}

\author{
Marta Hernandez
}

\section{Introduction : Question de méthode. Comment aborder Les Immatériaux?}

1 Je vais commencer cette intervention par aborder une question concernant la méthodologie pour l'analyse des Immatériaux aujourd'hui. Cette réflexion voudrait servir aussi à justifier, au moins dans une partie, le point de vue que je pris par rapport à cette exposition. Car s'il est clair que la méthode qu'on emploie lors de l'analyse d'un sujet ne va pas sans conséquences dans les conclusions, il m'a paru que cela était spécialement déterminant dans ce cas, dans la mesure où il s'agit d'un événement passé dont l'intérêt principal était d'en être spectateur.

D'où une contrainte qui n'est pas sans importance : l'analyste d'aujourd'hui sera obligé soit de travailler sans avoir jamais vu ce qui s'est passé en réalité au $5^{\mathrm{e}}$ étage du Centre George Pompidou du 28 mars au 15 juillet 1985, soit de se contenter d'un souvenir qui daterait aujourd'hui d'un peu plus de vingt-deux ans. Dans les deux cas, notre analyse souffrirait du même manque : celui du sentiment éprouvé par le spectateur lors de la réception de l'événement. Cela s'avère problématique malgré le caractère éminemment philosophique de l'exposition; celui-ci pourrait nous faire croire que les Immatériaux se laisseraient tout à fait comprendre à partir d'un discours. Mais, si d'un côté les Immatériaux ont la prétention explicite de rendre sensibles les enjeux théoriques de la "condition postmoderne", d'un autre côté tout discours a priori y ferait défaut, au sens où Lyotard dit dans un entretien accordé au périodique le Terminal le 01/09/1985 :

Nous avons choisi de présenter une dramaturgie pour que les gens soient "saisis dedans", qu'ils aient l'occasion de tirer le message dans le sens qui leur paraîtrait le plus propre. Le parti a été pris de faire sentir plutôt que d'enseigner. C'est que nous-mêmes sentons la situation, la conjoncture, plutôt que nous ne pouvons encore la penser.

3 D'ailleurs, même si la Postmodernité y servait de contexte théorique, de nombreux commentaires de Lyotard nous approcheraient de Discours, Figure, dans la mesure où il 
est question d'une sensibilité hors discours qui émergerait lors de la rencontre du spectateur et de l'œuvre, car, je cite Lyotard :

on ne prétend qu'à dégager une sensibilité habituellement refoulée » (recueilli par

le Quotidien de Paris dans un article du 28/03/ 1985).

Cependant, plutôt que d'aborder les Immatériaux dans son rapport à l'ensemble de la philosophie lyotardienne, j'ai préféré mettre en avant son propos interrogatif et orienter mon analyse dans cette perspective.

5 Aussi, puisque ce questionnement s'adressait au " grand public », je voulus lui trouver une place dans cette analyse. Mais quelle place? D'un côté il ne s'agissait pas de faire une analyse sur l'étude sociologique du public des Immatériaux. D'un autre côté l'idée d'inclure ce public dans ma réflexion afin de mettre l'accent sur l'incompréhension de l'événement par les visiteurs, me paraissait falsifier les choses dans un double sens: premièrement parce qu'on prend ce public comme s'il s'agissait d'une totalité homogène, alors qu'il y a eu différents types de public; et deuxièmement parce qu'entre la compréhension totale et l'absolue incompréhension il y a des degrés dus à des difficultés de différente échelle. Donc, je me suis limitée à utiliser cette « étude sur le public » des Immatériaux dans la mesure où je croyais pouvoir combler un peu le manque de mon absence, jouer un peu à faire comme si j'étais là.

6 Le fait de souligner qu'il s'agit d'interroger les visiteurs et non pas d'exposer une théorie veut servir à deux choses : 1) à ne pas forcer l'interprétation en vue d'atteindre une cohérence supposée entre la théorie et la pratique d'un auteur, parce qu'il s'agit d'un travail en équipe et parce que l'idée d'“auteur" était considérée comme un des préjugés modernes à mettre en question ; 2) à ne pas, en vertu de cette cohérence, refuser d'ores et déjà l'idée que les Immatériaux pourraient aussi interroger certains aspects de la philosophie lyotardienne. Je cite encore Lyotard:

Moi, j'ai été très déplacé par ce travail. La Condition Postmoderne n'était pas assez dramatisée, avait des issues trop simples; post-moderne, cela ne veut rien dire, mais on a quand même le sentiment qu'un idéal de clarté, de simplicité, un type de justification en cours depuis deux ou trois siècles n'est plus tenable. Post-moderne ainsi désigne un changement mais pas une période.

7 Donc je me suis bornée dans cette intervention à dégager certaines façons où les Immatériaux pourraient continuer à nous interroger. Vingt-trois ans après, destinataire longuement différée et sans recours à ma sensibilité pour juger de ses effets sur moi, j'ai travaillé sur les documents disponibles sur les Immatériaux. Ces documents sont : le catalogue de l'exposition accompagné de deux projets, «Les Épreuves d'écriture », les articles de presse que j'ai pu recueillir ; «L'étude de l'événement et de son public », la vidéo réalisée à l'occasion des Immatériaux (Octave au pays des Immatériaux), et l'œuvre collective « Modernes et après ».

\section{Présentation}

8 En effet, selon les deux projets de l'exposition qui ont été publiés, (et qui sont l'introduction du catalogue), les Immatériaux avaient l'objectif d'interroger les nouveaux matériaux du monde technique. Par le moyen de cette interrogation, ils visaient à éveiller chez le visiteur une sensibilité face à un nouveau monde qui, selon ses concepteurs, était à l'époque en train de naître. Dans ce monde nouveau il était question des nouveaux matériaux, de technologies nouvelles, de nouveaux modes de 
perception, de représentation et de symbolisation; de nouveaux moyens de décision, de conception et de production. Le premier projet, du 27 juin au 5 novembre 1984, disait qu'il s'agissait d'une «exposition expérimentale " parce qu'elle abordait « une analyse de phénomènes dont on commence tout juste à prendre conscience ».

On aurait tort de croire que ces phénomènes sont principalement des nouveaux objets technologiques. On n'a qu'à regarder un peu le catalogue pour se rendre compte que la plus part des œuvres et objets exposés n'avaient rien de nouveaux. Ceci a été la cause d'une gêne chez quelques critiques de l'époque. Michel Carnot, par exemple, auteur d'un des articles le plus sévère déclarait n'avoir trouvé là-bas que du « festival du déjà $v u$ » et « un magasin de curiosités naïf et macabre ». Pourtant ce « déjà vu » constitue l'une des singularités de l'exposition et de son énigme: le fait qu'on nous montre des extraits d'œuvres de Joseph Losey, Samuel Becket, Marcel Proust ou Henri Michaux pour illustrer ce nouveau monde technique qui déborderait les contours tracés par la pensée moderne, nous inviterait d'ores et déjà à ne pas adhérer à une réponse simple. À une réponse qui soit du type : les nouvelles technologies bouleversent tout, ou à son antithèse: les changements techniques ne touchent en rien l'essentiel; essentialité qu'on présuppose comme étant d'un tout autre ordre. Que l'on propose aux visiteurs de circuler sur un échiquier à l'échelle humaine, accompagné par un extrait de "Le scarabée d'or » d'Edgar Poe pour illustrer cette « révolution dans nos rapports avec la matrice ", montre un peu qu'il s'agit quelque part de déjouer nos opinions couramment acceptées. Opinions qui, conformément aux attentes, s'exprimeraient sous la forme « je suis d'accord» ou «je ne suis pas d'accord», «cela me plait» ou «cela ne me plait pas".

10 En réalité, dit Lyotard, ce n'est pas au niveau du contenu que les Immatériaux peuvent nous faire signe, mais en tant qu'exposition, c'est-à-dire par la façon qu'elle a de nous montrer les choses qu'elle expose. Sa valeur, contrairement aux expositions artistiques ordinaires, ne vient pas a priori de la notoriété de l'auteur ou des objets exposés, mais plutôt du travail d'organisation et de mise en forme de ces objets. Ceci rapprocherait les Immatériaux des expositions documentaires, construites autour d'un thème de réflexion qu'elles développent et illustrent dans un espace donné. Mais si les expositions documentaires ont pour but d'enseigner, d'apprendre quelque chose aux visiteurs et de leurs former une opinion, il s'agit pour les Immatériaux, je cite Lyotard, Le Monde du 3 mai 1985 de «faire regarder le déjà vu comme Duchamp faisait avec les ready-made, désapprendre le bien connu ». Dans plusieurs entretiens accordés aux journaux, ses principaux concepteurs, Lyotard et Thierry Chaput, soulignent que son caractère expérimental et non pédagogique fait partie de l'enjeu majeur de l'exposition. Ils précisent qu'il s'agit d'« une exposition à questions et non à définitions", et ils reconnaissent qu'elle n'est « ni explicative ni complète».

11 Donc, il ne s'agit pas pour les visiteurs d'apprendre mais plutôt de désapprendre ce qu'ils croyaient savoir. Et pour atteindre ce but les Immatériaux proposent non pas une explication nouvelle des phénomènes mais une dramaturgie au sens théâtral. Dramaturgie d'un corps à la limite de sa présence où l'espace n'est pas découpé par son rapport à l'homme. Mais c'est plutôt la place du corps humain qui est déstabilisée par un espace dont il n'est plus la mesure et qui le déborde. Hommage à Samuel Beckett, dans ce site du "Théâtre du non corps » où sont présentées des situations inventées, inspirées des pièces de l'auteur à la limite de la présence du corps. Celui-ci est réduit par exemple à une lumière sur une bouche, où les personnages sont dans l'ombre et 
n'apparaissent dans la lumière que lorsqu'ils parlent. Il s'agit pour Lyotard, je cite, dans un entretien accordé à CNAC Magazine le $1^{\mathrm{er}}$ mars 1985, « Des personnages soumis à la règle du langage et non de la présence corporelle.»

$\mathrm{Du}$ "théâtre $\mathrm{du}$ non corps» inspiré par Becket au «labyrinthe du langage » d'inspiration borgésienne, c'est sur le langage et l'espace indiscernablement que les Immatériaux veulent nous interroger. Notre rapport au « matériau » en tant que support d'inscription d'un code serait à la fois et de l'espace et du langage. Ils portent cette interrogation principalement sur la sensibilité des visiteurs en les destituant de leur place "privilégiée » de spectateurs, pour les immerger dans un espace labyrinthique. C'est ainsi que, dans cette espace labyrinthique sans « Minotaure, sans Monstre et donc sans saveur » (Libération), le fait de prendre place (de se situer ou de se retrouver dans cet espace), et de prendre parole (de manifester son avis sur le sujet de l'exposition ou sur l'exposition elle-même) revient au même acte "difficile» ou "impossible». Situation qui met en évidence le malaise éprouvé par un individu placé dans une position intenable.

13 Je crois que cette "position intenable » du destinataire des Immatériaux, relève du fait que d'un côté on postule une sensibilité égale en tous et chacun en les présupposant capables de produire un sens, et d'un autre côté on met en jeu un système de règles qui n'est pas accessible à tous de la même façon et que renforceraient les inégalités effectives. C'est là ce qui constitue l'arrière-plan de l'exposition, où je trouve qu'il y a un enjeu majeur pour la pensée sur la technique. Cet enjeu est le suivant: le destinataire d'un message, pour être en mesure de produire une réponse à la demande du destinateur, doit posséder la maîtrise du code (de la langue) dans laquelle ce message lui parle afin de le déchiffrer. Ce code est à la base du partage d'un savoir. Dans cette perspective, une langue (un code) n'est principalement ni un simple moyen de communication ni quelque chose que l'on puisse se contenter de qualifier suiréférentielle (au sens où elle ne serait référée à rien d'autre qu'elle-même), mais partagée, de façon consciente ou inconsciente et le plus souvent de manière inégale.

14 «L'étude de l'événement et de son public » met en évidence cette inégalité à l'égard de l'incompréhension. Compréhension ou incompréhension qui commence par la sensibilité dans la mesure où il s'agissait premièrement de se situer dans un espace et de se faire une idée sur son fonctionnement; et cela par des tâtonnements, par des essais, et au fur et à mesure qu'on parcourait cet espace, qu'on participait au jeu des Immatériaux. En l'absence des explications à propos de l'exposition et de repères chronologiques et thématiques, une question devrait émerger dans chacun des visiteurs et différemment selon le site de l'exposition dans lequel ils se trouvaient: celle du Comment ça marche? Question à la lumière de laquelle on comprend pourquoi, je cite le quotidien Libération du 28 mars 1985 :

$\mathrm{Ni}$ vraiment scientifique, ni tout à fait artistique, l'exposition de Jean-François

Lyotard est, à proprement parler, technique.

Donc, d'une part il y était question de la liberté des visiteurs. Pour cela, la disposition spatiale des Immatériaux leur permettait de choisir librement leurs parcours et de quitter l'exposition à tout moment quand ils le désiraient. Mais d'une autre part il était aussi question d'un pouvoir; pouvoir exercé sur les visiteurs qui étaient saisis à l'intérieur de cet espace. Espace qui les poussait à jouer d'une certaine façon dont ils n'étaient pas a priori censés connaître les règles. Il s'agissait alors de jouer, sans savoir à quoi cela allait les conduire. C'est par cette démesure entre la complexité du jeu et 
l'ignorance de la plupart des visiteurs à l'égard de son fonctionnement qu'on a parlé d'un malaise éprouvé par la grande majorité ; en gros, le public ne se sentait pas à la hauteur de produire une réponse conforme à ce qu'il croyait qu'on attendait de sa participation. Comme conséquence de cet excès de l'exposition on a signalé l'autoexclusion d'une partie du public (de son refus à jouer) et la sous-utilisation de certains sites de l'exposition.

\section{Les Immatériaux et l'espace culturel}

Cette introduction voudrait nous conduire vers l'idée que les Immatériaux en tant qu'exposition peuvent fournir un modèle pour penser l'espace culturel. Ainsi compris, l'espace culturel est un support d'inscription où est inscrite une pluralité de codes. À l'opposé d'un espace géométrique un aspect individuel/sensible y est sollicité. Cet aspect d'interaction entre la matrice d'un code et le destinataire a été suggéré dans le site «Variables cachées ", je cite le catalogue :

À travers une enquête sur le visiteur est révélée une variable cachée de l'exposition: le public. Recherche (in situ) de l'ensemble des règles (matrice) commandant le phénomène "visite des Immatériaux ». Impossibilité de saturer la matrice, d'isoler le système du contexte.

17 Cette présentation du public comme variable cachée (d'un système, le système de la culture) qui interagit avec la matrice est à son tour mise en rapport avec le site « irreprésentable » appartenant à la zone "matière ». "Irreprésentable » est dit à propos de l'impossibilité de restituer visuellement la matrice d'un élément quand il est soumis à quatre variables ou plus. C'est la croisée de ces variables comportant un aspect généralisant et un aspect individuel qui serait la forme de base de l'espace culturel. Forme en chiasme qui n'est pas représentable dans un espace cartésien. Carrefour d'intensités qui constitue la forme dynamique du partage de la culture.

Ce partage, avant d'être celui du sens dont chacun serait censé créer le sien (en fonction de sa maîtrise du jeu, de son envie de jouer, du choix plus ou moins hasardeux de son parcours, du temps dédié à l'exposition, etc.) serait celui d'un pré-sens (comme un pré-sens de l'orientation) dont l'exposition elle-même fournirait un modèle de milieu. Ce milieu du pré- sens qui serait la condition de possibilité, aussi bien que d'impossibilité du sens créé par chacun, à la différence d'un a priori subjectif au sens kantien, comporterait une démesure entre l'aspect aprioristique généralisant et l'aspect individuel/sensible.

19 C'est parce qu'il y a une non réductibilité entre ces deux aspects que le sens est aussi conçu comme une dynamique : il est un parcours, une trajectoire, sans une véritable fin. Dans cette perspective la fin n'est pas l'achèvement de quelque chose mais une limite non préfixée dont on peut s'approcher ou s'éloigner, qu'on peut accélérer ou ralentir. Donc plutôt que de trouver cette fin comme si l'on allait révéler le mystère du labyrinthe, il s'agissait pour les Immatériaux de jouer avec elle, chaque visiteur décidant de son parcours. Parcours initiatique, comme tout labyrinthe, mais où le début et la fin ne sont pas les extrêmes qui cernent le sens, quelque chose comme les bouts d'une définition, mais deux points de repères aussi hasardeux (et nécessaires) que le parcours lui-même. Cette idée était mise en jeu par l'absence des commentaires explicatifs de l'exposition, et leur remplacement par un casque individuel sonore qui avait pour but 
de créer un effet de déstabilisation et d'isolement. D'où cette contradiction d'un univers bondé mais en même temps « déshumanisé ".

De là une analogie possible avec l'univers kafkien sollicité par quelques visiteurs qui comparaient les Immatériaux avec l'exposition sur Kafka, laquelle avait eu lieu à Beaubourg peu de temps avant. Pour donner une piste sur ce qui pourrait créer cette analogie avec l'espace kafkien (espace qui est aussi à la croisée de l'absurde et de la nécessité de la loi). Je cite Didi- Huberman, dans son analyse du Procès :

Une trame singulier d'espace ouvert et clos en même temps. Une brèche dans un mur, ou une déchirure, mais ouvrée, construite, comme s'il fallait un architecte ou un sculpteur pour donner forme à nos blessures plus intimes. Pour donner, à la scission de ce qui nous regarde dans ce que nous voyons, une espèce de géométrie fondamentale. » Et plus loin «Il (Kafka) sait aussi que tout cela est une géométrie, c'est-à-dire un jeu de la forme, un jeu de construction, une ironie construite sur la fin.

21 Pourtant, s'il est question d'une géométrie fondamentale, d'une proto-géométrie dans la matrice, l'espace culturel, on l'a vu, n'est pas moins dominé par un aspect individuel. Autrement dit, la forme projective de l'exposition, forme labyrinthique, ne saisit pas la forme dynamique de base qui commande la participation des visiteurs. C'est par cet aspect individuel que les Immatériaux réclament une action; ils veulent être le déclencheur d'une action dans l'absence de réponse à la question Qui ? C'est qui l'auteur des messages que je reçois? qui est-ce qui me commande de jouer d'une certaine façon? c'est qui le producteur de cette loi ? Cette question restait aussi sans réponse dans la littérature kafkaïenne et aussi dans celle de Becket. Mais chez ces deux auteurs, cette loi mystérieuse du pouvoir condamnait les personnages, du Procès ou de En Attendant Godot, à la paralyse, à l'attente infinie, à la forme d'un boucle. Dans la mesure où les Immatériaux réclament une action sans donner de directrices préalables, ils rendent du même coup la question du qui inappropriée, non-opérative. Et ceci, soit parce qu'elle ne nous amène qu'à l'attente, à l'inaction, soit parce que dans l'époque contemporaine cette question est devenue obsolète. Voir par exemple le site "Tous les auteurs" appartenant à la zone "maternité ", et aussi les "Épreuves d'écriture ", fruit d'un processus d'écriture semi-collective qui consistait à demander à un certain nombre d'intellectuels, reliés par ordinateur, de rédiger des définitions et de dialoguer autour d'une liste de 50 mots relatifs à la problématique de l'exposition, proposés par Lyotard.

Les Immatériaux substituent la question technique du Comment ça marche, une question qui réclame en elle-même la participation active du destinataire, à la question de l'autorité. L'émergence de ce questionnement par le fonctionnement déplace la question du Qui ? ou à Qui ? créant un effet de brouillage sur les dichotomies objectif/ subjectif, généralisant/individuel par rapport à l'espace culturel. La question sur le fonctionnement fait appel au milieu qui met en place ces couples d'opposés. Dans ce sens ce milieu serait ce qui fait marcher et l'objet et le sujet dans leurs rapports dynamiques.

Platon lui-même dans le Timée traite une alternative qui dépasse cette dichotomie avec l'idée de khôra. L'existence de la khôra résume chez Platon un double dilemme intellectuel: d'un côté elle justifie la différence ontologique du sensible et de l'intelligible ; et d'un autre côté elle esquisse une réponse à l'idée que si l'espace existe, il doit être contenu dans un autre espace et ainsi de suite. Ce dernier aspect constitue aussi une approche possible de l'idée lyotardienne d'espace figural dans la mesure où un espace ainsi considéré devient indéfinissable pour toute abstraction philosophique. 
Cet espace au sens de khôra qui n'est rien de concret, et dont on ne peut pas dire qu'il est ceci ou cela, nous empêcherait de définir l'espace culturel de façon réaliste, c'est-àdire comme un milieu qui unit des objets existant réellement. Et au contraire, il ne peut pas être non plus compris d'une manière idéaliste «kantienne " comme une forme abstraite réunissant ce qui est concret. Il comporterait à la fois un aspect géométrique/ langagier et un aspect individuel/sensible. Cet «à la fois " n'est pas une synthèse de contraires, mais plutôt un carrefour d'intensités dont le ressort est de l'ordre de la non mesure, de l'excès, de la non conciliation. De même que Lyotard parle d'une "sensibilité refoulée » issue d'une démesure entre un aspect langagier et un aspect sensible.

La rencontre de ces deux axes a lieu dans un espace (dans un milieu) qui est leur matériau, leur support d'inscription. L'espace compris comme un support, dont l'exposition elle-même fournit un exemple et qui «remet en cause la présentation traditionnelle des expositions héritières des salons du XviII ${ }^{e}$ siècle et des galeries ", interroge alors plus généralement sur une certaine idée de l'espace : celle qui veut faire de l'espace quelque chose de séparé par rapport au sujet et aux phénomènes. Propos qui pour garder la cohérence avec le discours lyotardien de la postmodernité peut être compris aussi comme une certaine critique de la «modernité cartésienne ». En gros, ce que Descartes énonçait avec la distinction qu'il met entre res cogitans et res extentia était l'indépendance, au moins de droit, entre la pensée et l'espace, entre l'esprit et la matière.

Cette distinction est déjà mise en question par le schème qui constitue un des arrières plans de l'exposition: celui du modèle communicationnel emprunté à la linguistique pragmatique dont les représentants principaux seraient $\mathrm{H}$. Laswell et $\mathrm{R}$. Jakobson, qui considère tout objet général ou tout phénomène comme un message, c'est-à-dire comme un ensemble de signes.

Ce schéma est le suivant : tout message est indissociable du support où il est inscrit. Ce support est le «matériau» du message. Le matériau n'est pas la matière. Le matériau est le moyen par lequel un message est destiné. Il serait ce "de" quoi les phénomènes ou les messages sont faits et à la fois ce "en" quoi ces phénomènes se donnent à voir, y prennent place. Le support, le matériau, est parti constitutif du message dans la mesure où il est le support d'un code. Ce code ou langage en général, est inscrit dans le support comme la distribution réglée d'éléments discrets qui constituent le matériau. Cette distribution ou disposition de tout matériau en général en éléments discrets s'oppose à l'espace cartésien défini essentiellement comme un espace continu (non réglé) et homogène, qui est l'espace géométrique euclidien. Cet espace continu et homogène qui est le support d'inscription des corps géométriques se caractérise par son indépendance par rapport au sujet et aux objets. Il est la substance étendue distincte de la substance pensante et pour cette raison elle peut être isolée, objectivée et considérée comme objet de la connaissance du sujet. Par contre dans ce schéma la matériau disparaît comme entité indépendante au sens où il n'est pas séparable d'un code, ce même code qui constitue aussi les messages. Support et messages partagent le même code, ou matrice. Donc, pour les déchiffrer il faut faire un peu comme dans un «Jeu d'échecs » (Site de l'exposition appartenant à la zone « matrice ») je cite :

Remonter à partir d'une suite d'événements apparemment fortuits jusqu'à la règle $\mathrm{du}$ jeu (matrice) qui la concerne. Un événement n'a lieu que s'il est permis par la matrice (par exemple, l'ensemble des règles lexicales et syntactiques du jeu d'échecs) et par la situation ou contexte, (l'état du jeu à un instant donné.) 


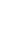

un détour par la métaphysique platonicienne. Dans le Philèbe on trouve l'idée d'un "rapport de famille» entre l'âme humaine et la vie intellectuelle, comme une disposition semblable à deux choses apparemment hétérogènes. C'est cette disposition commune qui fait appartenir l'une et l'autre au même genre. Ainsi, Platon dit que «L'intellect est parent (бuүyعvńs) de la cause et rentre plus ou moins dans le même

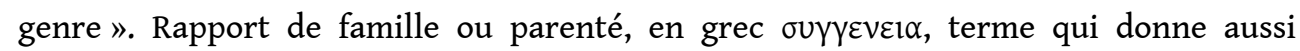
"semblable ». C'est ainsi qu'au début de ce dialogue où Socrate introduit la discussion autour de la recherche du bon ou de la vie heureuse, à partir de deux alternatives opposées celle du plaisir et la jouissance et celle de la sagesse et l'opinion droite, il dit qu'avant de se décider pour l'une ou l'autre il faut savoir quelle est la disposition de 
l'âme humaine. Si la vie heureuse est celle de la sagesse c'est parce que l'âme a un rapport de parenté plus proche avec l'intellect qu'avec le plaisir. Donc, c'est parce qu'elle entretient un rapport de famille avec la sagesse qu'elle est semblable à celle-ci et que le bon sera la sagesse.

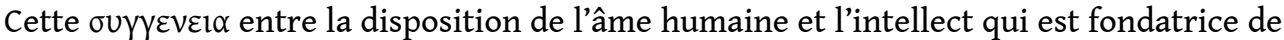
la vie heureuse pour tous les hommes, est aussi la forme de base de l'anamnèse, de la mémoire ou du «faire mémoire ». L'anamnèse, issue des mots grecs ána (remontée) et mnémé (souvenir), serait le souvenir du récit des antécédents (des parents ou des

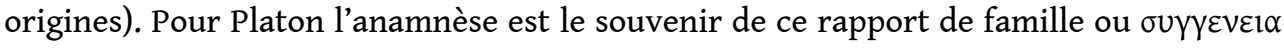
entre l'idée et l'âme humaine avant l'incarnation de l'âme dans le corps ; souvenir qui resterait inconscient sans l'opération de la réminiscence.

Ce détour par la métaphysique platonicienne veut être une voie pour comprendre la forme expérimentale de l'exposition en tant qu'elle aussi relève d'une anamnèse. Je cite Lyotard, «Un travail » dans L'étude de l'événement et son public:

Une forme indéterminée, insaisissable par concept, vers laquelle le sentiment seul, s'il est interrogé, épié, (c'est l'anamnèse), apuré, nettoyé des intérêts fantastiques et autres, peut guider en faisant discerner les moyens qui ne la traduiront pas. Singulière fidélité, probité à l'endroit de quelque chose qui n'est pas déterminée.

Cette anamnèse dont parle Lyotard n'est pas alors l'anamnèse d'une forme déterminée,

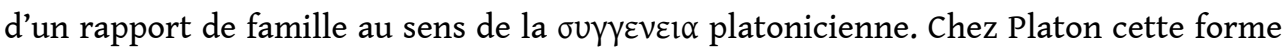
commune aux idées et à l'âme humaine serait le fondement pour penser une mesure commune à l'une et aux autres. Par contre les Immatériaux tentent de "faire mémoire » d'un autre rapport de parenté. Cette parenté alors ne sera pas à l'origine d'une mesure commune mais d'une non mesure: d'une forme indéterminée, de quelque chose qui

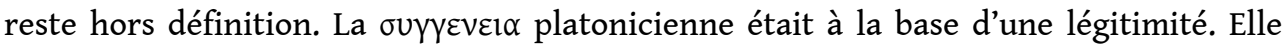
faisait de la vie intellectuelle la vie la plus heureuse conformément à la disposition de l'âme, et dans ce sens la vie la plus légitime conformément à la norme. Par contre, le rapport de famille dont il est ici question de faire anamnèse serait plutôt le résultat d'un accouplement illégitime, d'une hybridation.

L'exposition est en elle-même un genre hybride. Elle est un mélange de genres qui ne font pas une synthèse. Dans ce sens les Immatériaux ne sont pas une exposition sur les expositions en général. Elle crée son propre objet et elle le fait par un nouveau genre d'exposition hybride : ni documentaire, ni artistique, ni exposition à spectacles, etc. mais et documentaire, et artistique, etc. à la fois. Par cette hybridation les Immatériaux opèrent un effet de brouillage des catégories traditionnelles. C'est par cette hybridation qu'elle produit un flou, une illisibilité, d'où surgit une interrogation.

On trouve cette idée d'un genre hybride dans le Timée de Platon. Ainsi, Khôra serait un genre bâtard issu d'un mariage illégitime : celui du sensible et de l'intelligible. Raison pour laquelle Khôra se soustrait à l'anamnèse platonicienne et reste alors hors de la connaissance. Sa forme indéterminée est plutôt entrevue comme dans un rêve, de façon floue, insaisissable par sa forme dont on ne peut pas dire qu'elle est ceci ou cela, je cite Platon, Le Timée :

[...] il y a toujours un troisième genre, celui du lieu (khôra) : il ne peut mourir et fournit un emplacement à tous les objets qui naissent. Lui-même, il n'est perceptible que grâce à une sorte de raisonnement hybride qui n'accompagne point à la sensation: à peine peut-on y croire. C'est lui certes que nous apercevons comme en un rêve, quand nous affirmons que tout être est forcément quelque part, 
en un certain lieu, occupe une certaine place, et que ce qui n'est ni sur la terre, ni quelque part dans le Ciel n'est rien du tout.

L'anamnèse tentée par les Immatériaux est alors la mise en forme d'une hybris dans le double sens grec de démesure et de mariage illégitime, insaisissable par la forme d'une définition mais susceptible d'être rendue visible/sensible par les formes artistiques. Je pense que c'est ainsi que les Immatériaux mettent en question certaines idées exposées dans la Condition postmoderne, qui sont visiblement retravaillées dans La Postmodernité expliquée aux enfants, je cite, p. 113 :

Tu comprends qu'ainsi compris, le « post- » de « postmodernité » ne signifie pas un mouvement de come back, de flash back, de feed back, (c'est-à-dire de répétition) mais un procès en "ana- ", d'analyse, d'anamnèse, d'analogie, et d'anamorphose, qui élabore un « oubli initial».

Élaboration d'un oubli initial qu'il faut comprendre dans le sens d'une "perlaboration", néologisme inventé pour traduire le terme allemand Durcharbeitung que littéralement l'on traduirait par un «travail à travers ». Cet «à travers » comprend le double mouvement de création et de révélation, qui est fondateur d'un nouveau plan pour la pensée. Ce plan ou agencement se projette vers l'avenir (et dans ce sens il a une valeur d'initiation en lui-même) et vers le passé au sens où il crée un nouveau rapport aux origines.

Les Immatériaux, traduisent dans sa mise en forme ce travail de perlaboration, d'enchaînement de scènes dont le sens réside non pas dans les objets exposés mais dans l'enchaînement même. Il s'agit d'un pré-rapport qui porte le sens, avant la production de sens par les visiteurs lors de leur parcours. C'est par cette dynamique double de création d'un sens individuel et de révélation de ce qui était déjà donné, que l'a travers du travail artistique dépasse les dichotomies subjectif/objectif, de l'individuel et du généralisant, du langage et de la sensibilité.

4 Ce dépassement par la forme appelle au contenu de l'exposition dans la mesure où cet enchainement fait des objets exposés des nuances d'un sens qui circule entre eux en les traversant. Ces objets fonctionnent comme un à travers qui dévoile ce rapport de parenté hybride, origine d'une démesure, entre l'esprit et la matière, entre le langage et la sensibilité. Si d'un côté cette non mesure entre le langage (généralisant) et la sensibilité (individuel) a comme conséquence le refoulement de la sensibilité, d'un autre côté cette hybridation ou union illégitime de la matière et de l'esprit serait l'objet d'un oubli en lui-même.

Cet oubli s'énonce pour l'ensemble de l'exposition par les questions -mât (d'où Matériau, Matrice, Matériel, Matière, Maternité) qui sont explicitées selon le modèle communicationnel de la pragmatique linguistique. L'objet de l'anamnèse porte dans une grande mesure sur la remémoration de la racine -mât, du sanscrit, qui donne mātrā (mesure, quantité, degré, ordre, division minime ou atome ; mais aussi «matière", choses matérielles, biens, moyens de subsistance) et mātrka (mère, maternel ou origine et dans ce sens matrice). - Mât révèle une origine étymologique commune de la mère, de la mesure et de la matière, dont la khôra de Platon fournirait encore un exemple parce qu'elle est la mère, la nourrice ; le matériau avec lequel le démiurge fabrique le mode sensible et le crible qui sépare les quatre éléments par des secousses, comme celui d'un agriculteur qui sert à séparer le bon grain du mauvais.

Cette indécision à l'égard de la khôra (et la mère et le matériau et le porte empreinte et le milieu spatial) aurait un analogon dans les Immatériaux dans la mesure où ils 
traduisent aussi un concept qui est contradictoire en lui-même, celui du matériau immatériel. Quant à la création du nom c'est un néologisme qui substantifie de manière inusitée une forme d'adjectif (immatériel) ou qui confère à un substantif ordinaire (matériaux) une forme négative. Cette indécision aussi à l'égard de son nom, fait des immatériaux l'enjeu d'une indiscernabilité, d'une frontière floue et imprécise qui pourrait s'expliciter dans cette question : comment alors tracer une distinction claire et nette entre l'idée d'un matériau immatériel proposé par l'exposition comme l'ouverture d'une nouvelle époque et l'immatérialité qui participe déjà à la constitution du matériau au sens de la khôra platonicienne? Car si Immatériau n'est pas le contraire de matériel mais du matériau; et si un matériau, n'est pas la matière, mais le support, porteur de sens lui-même, tout matériau ainsi considéré aurait les traces, les marques d'une immatérialité, d'une intelligibilité. Dans ce sens la khôra de Platon, matériau invisible et informe, serait un exemple d'Immatériaux. C'est dans ce sens que l'enchaînement élaboré par les Immatériaux, celui qui conduit à l'anamnèse, peut être pris pour un exemple d'enchaînement à partir d'un différend. Ce différend, hybridation de la matière et de l'esprit, était quelque chose dont on supposait l'existence sans pouvoir en témoigner. Ce qui apporterait alors les nouvelles technologies, serait cet "à travers " du travail qui rend visible (et dans ce sens permet d'en témoigner) quelque chose qui restait relativement caché : ainsi les nouvelles technologies « révèlent que l'esprit de l'homme [...] est une partie de la «matière " qu'il projette de maîtriser et que convenablement traitée, la matière peut s'organiser en machines qui supportent avantageusement la comparaison avec l'esprit. De l'esprit à la matière, la relation n'est plus d'un sujet intelligent et volontaire à un objet inerte. Ils sont cousins dans la famille des « immatériaux ».

\section{Les Nouvelles technologies et la destinée en chômage?}

Pourtant, par rapport au rôle des nouvelles technologies dans les Immatériaux, je trouve qu'il y a une certaine ambiguïté dans le propos de l'exposition. En partie je crois que cela s'explique par le fait qu'en principe il s'agissait pour Lyotard d'un travail de commande. Il a pris la direction théorique du projet une fois que le sujet lui a été proposé. Ce sujet qui a été inscrit au programme du CCI s'appelait «Matériaux Nouveaux et Création », je cite Lyotard :

Lorsqu'on m'a demandé de venir pour des raisons que je continue d'ailleurs d'ignorer, c'est ce qu'on m'a proposé. J'ai évidemment refusé parce que je ne connais rien aux matériaux, que «nouveau » ça ne veut rien dire et que « création » c'est de la théologie ou de l'esthétique romantique, c'est-à-dire des choses que précisément il faut mettre en question. On a insisté néanmoins pour que je m'en occupe en me disant que je pouvais orienter le projet comme je le voulais. (Libération du 28 mars 1985)

Puis il y eu un premier projet. Ce premier projet sous la direction de Lyotard s'appelait "La matière dans tous ses états ». Mon hypothèse c'est que de "Matériaux Nouveaux de Création " passant par « La matière dans tous ses états » aux Immatériaux, il y a eu un déplacement du contenu de l'exposition vers un travail beaucoup plus artistique et un arrière-plan philosophique qui excédaient l'idée de départ. Il me parait pourtant que des résidus de ces deux premiers projets ont subsisté de manière à rendre difficile une explication tout à fait cohérente du propos de l'exposition, surtout du rôle joué par les 
nouvelles technologies dans le changement d'époque. Comme preuve de cette ambiguïté, nous pouvons mentionner le fait que cet accent mis sur la forme de l'exposition n'a pas été toujours clarifié avec la même précision. Et quand Lyotard parle du contenu des Immatériaux il apparait que parfois il considère ces Immatériaux comme ayant une existence objective, comme étant des produits des technosciences. Des « nouveaux matériaux » qui seraient en fait des Immatériaux parce qu'ils sont engendrés par l'informatique ou l'électroniques, je cite: "Des nouveaux genres apparaissent reposant sur les nouvelles technologies». Les nouvelles technologies, qui sont des « substituts d'opérations mentales et non plus physiques. ».

En ce qui concerne cette dernière distinction entre mental et physique, je ne vois pas pourquoi d'un côté on nous dit que l'esprit et la matière sont cousins de la même famille, et d'un autre côté on fait reposer la rupture avec la modernité sur une distinction radicale entre les opérations mentales et les opérations physiques. Alors que dans la perspective d'une perlaboration on pourrait très bien soutenir que toute opération mentale, tout calcul, est quelque part une opération de la matière. Et au contraire, on pourrait très bien soutenir que tout appareil de mesure, même le plus primitif est le substitut d'une opération mentale. C'est pour cela que j'ai préféré rester avec l'idée que c'est dans sa façon particulière de "travailler à travers " qu'une technologie, ou une technique, peut élaborer quelque chose et par rapport au passé et par rapport à l'avenir : un avenir qui doit comprendre quelque chose d'un être déjà là ; et un passé qui élabore ce que nous sommes aujourd'hui.

Il y a encore une question qui n'est pas facile à concilier avec l'idée générale que je me fais de cette exposition. Je parle de la perte du destin de l'humanité, thème référé dans quelques sites de l'exposition et de son rapport avec l'immatérialité du support. Ainsi, dans le vestibule d'entrée de l'exposition le visiteur trouvait un bas-relief égyptien, fragment de paroi du temple de Karnak-Nord, où apparaît la scène de la déesse offrant le signe de vie à Nectanébo II. À la bande-son il y avait du souffle. À propos de ce site on lit dans le catalogue :

Les humains recevaient la vie et le sens: l'âme. Ils devaient la rendre, intacte, perfectionnée. Y-a-t-il aujourd'hui quelque chose qui leur soit destiné? C'est un enjeu majeur de cette manifestation.

Dans le vestibule de sortie la même paroi était présentée, projetée en image tremblée, grâce à laquelle l'effet-matière s'absente. À la bande-son, le silence. Je cite le catalogue :

La vie, le sens, nous sont-ils encore donnés (destinés) par une Mère, comme un message à déchiffrer, à honorer et à lui restituer une fois venue à l'échéance ? Notre façon de les recevoir, de les connaître, de les transformer, de les représenter, de nous en acquitter n'inflige-t-elle pas un démenti à l'antique conviction que l'âme devait être rendue, intacte, perfectionnée? Sinon un désaveu, du moins, un trouble?

Cette idée d'utiliser la même image différemment traitée à l'entrée et à la sortie, alors qu'on soulignait la liberté des visiteurs dans leurs parcours, m'a fait plus l'effet de la clôture d'un sens que d'un questionnement.

Par le contexte postmoderne de l'exposition je comprends bien qu'il s'agit aussi d'interroger le sens de l'histoire selon la pensée moderne; histoire qui en gros est conçue dans la perspective d'un progrès illimité de l'humanité en tant que sujet rationnel. Mais ici on nous parle des antiques, et on nous parle d'une idée de "destin " qui n'est pas celle de la modernité. Est-ce qu'on veut nous dire que cette idée d'histoire comme progrès, serait-elle débitrice du destin tel qu'il était pensé par les antiques, 
voire par les égyptiens, par les grecs? Je trouve très difficile, sans une analyse plus approfondie, de répondre à cette question affirmativement.

51 Je m'accorde à l'idée qu'une pluralité de codes, rendue plus évidente par la multiplication de langages technologiques, met en question l'idée d'une matrice universelle vers laquelle on pourrait remonter et déduire l'origine du sens (de l'humanité, de l'histoire). Dans l'absence de cette matrice universelle, parler d'une histoire ou d'un destin universel, n'a pas beaucoup de sens, ou ne résout pas grande chose. Mais, cette idée de la perte du destin est mise en rapport premièrement, non pas avec la matrice, mais avec le matériau. Je cite le catalogue :

Matériau : ce sur quoi s'inscrit un message : son support. Il résiste. Il faut savoir le prendre, le vaincre. C'était le métier, faire une table avec arbre. Qu'arrive-t-il si l'on conçoit, simule et réalise le matériau selon la nature du projet ? Toute résistance au projet d'inscrire un message serait vaincue. Le message ne rencontre pas son support, il l'invente. Le travail n'affronte pas son objet, il le calcule et le déduit. [...] La question pressante : avec la perte du matériau, la destinée en chômage ?

Pour apprécier un peu la portée de cette question, je vais faire appel encore une fois à l'étymologie: moïra en grec "destin » signifie en même temps "part", "portion", "partie d'un terrain ». Elle est aussi l'unité de mesure de l'angle d'un triangle. Cette pluralité d'acceptions qui traduit la conception grecque du destin (la moïra est la loi de partition qui impose à chacun une part de bien et de mal) montre aussi l'origine commune du destin et de la mesure liée à la répartition de la terre. Ce rapport est accentué par le fait que la géométrie égyptienne, dans son sens primitif de mesure de la terre, est née pour résoudre un problème pratique: celui des débordements périodiques du Nil. Ainsi, la mesure permettait une distribution de la terre selon l'ordre établi avant le débordement du fleuve. Dans cette perspective la terre est un support, un matériau, qui permet l'inscription selon la mesure. De cette façon la mesure de la terre était aussi pour les égyptiens la condition de possibilité de la conservation de l'ordre social (et du destin de chacun) selon les hiérarchies. Elle était le support d'inscription d'une mémoire collective. Or, dans le Timée, Platon s'approprie le souci d'une possible destruction de la terre (destructions qui avaient lieu périodiquement dans la Grèce antique) dont les débordements du Nil lui fournissaient un exemple. Le rapport support-destin répond dans le Timée à une double exigence dans la mesure où il est impliqué par la khôra 1) il est de l'ordre de la nécessité. Khôra est la nécessité, dans le sens où elle est éternelle et aussi dans le sens grec d'óvóykń qui signifie en même temps «résistance » ou ce qui oppose une résistance. 2) Parce qu'elle résiste à ces destructions de la terre, elle peut être le support d'inscription d'une mémoire collective. Elle résiste aussi dans un double sens : 1 ) parce qu'elle est immatérielle elle reçoit toutes les formes intelligibles sans prendre jamais la forme d'aucune d'elles (dans ce sens elle est informe) ; 2) Parce qu'elle est éternelle, antérieure à la naissance du temps, elle n'est pas soumise à la corruption. Khôra est donc un matériau immatériel, un Immatériau. Si elle était matérielle, susceptible de destruction, comme la terre, comme les choses sensibles, elle ne pourrait pas être le support de la mémoire collective, du destin du peuple d'Athènes, telle qu'il était pensée par Platon. S'il en est ainsi, en quoi les Immatériaux changeraient notre rapport au destin au sens de Lyotard? Est-ce que l'immatérialité des matériaux ne peut pas être pensée comme une résistance? Comme une résistance à la fin. Est-ce que cette incroyance à l'égard d'un destin de l'humanité ne vient pas aussi de la conscience que le monde peut finir bien avant que l'être humain puisse accomplir quelque chose comme son projet dans le monde ? Est-ce 
que ces Immatériaux ne sont pas traversés d'un certain espoir de transcendance? Transcendance de cet espace-ci dans un autre espace.

Dans ce sens et pour finir, je cite Derrida, Les épreuves d'écriture, à propos du mot Dématérialisation:

La matière n'est plus un support, la substance, le sujet, le terme aux confins d'une opposition. Non plus un réceptacle, ni une matière intelligible (hylè noété) ou un « incorporel ». Khôra peut-être (espacement, emplacement au-delà des oppositions, tertium quid pensé " comme en rêve »). Insensible. Capacité de résistance (restance) plus intraitable que jamais (non opposable) : la mort inéluctable dans mon rapport à moi, ce que je m'envoie sans support apparent, à une vitesse absolue, avec toi, avec moi, dans le pays.

\section{AUTEUR}

\section{MARTA HERNANDEZ}

Doctorante, université de Madrid/Paris 8 